\title{
Fuzzy self-tuning PID on The Liquid State Ammonia Nitrate Storage Tank temperature control system
}

\author{
WANG Yue-sheng ${ }^{1, a}$, QU Jun-qiu ${ }^{2, b,{ }^{*}}$ \\ ${ }^{1,2}$ Institute of Automation, Hangzhou Dianzi University, Hangzhou Zhejiang 310018, China \\ awys@hdu.edu.cn, bqujunqiu@foxmail.com \\ ${ }^{*}$ Corresponding author
}

\begin{abstract}
In the current Liquid State Ammonia Nitrate Storage Tank System, the traditional PID temperature control is used mostly. By controlling the flow of heating steam valve, the outlet temperature of the liquid state ammonia nitrate storage tank can be controlled. However, during the production process, much interference exists with the characters of nonlinear, time-varying and large lag which the controlled objects have. Therefore, the single PID parameter cannot control the temperature accuracy. During the operation of the entire production line, a relatively stable temperature of the production process should be maintained, while the excessive consumption of energy in the production process should be reduced. For this situation, the fuzzy PID control is a better choice for temperature control. According to the difference of temperature departure and its rate of change, the fuzzy parameters self-tuning PID algorithm can control the system by choosing different P, I, and D parameters in order to achieve the online self-tuning function for better using in the operation of the product line.
\end{abstract}

Keywords: Liquid ammonia storage tank, Fuzzy self-tuning,PID, Temperature control.

\section{基于模糊自整定PID温度控制的液态硝铵储罐系统 \\ 王越胜 ${ }^{1, a}$,曲俊秋 ${ }^{2, b},{ }^{*}$ \\ ${ }^{1}$ 杭州电子科技大学自动化研究所, 浙江杭州, 中国 \\ 2杭州电子科技大学自动化研究所, 浙江杭州, 中国 \\ awys@hdu.edu.cn, bqujunqiu@foxmail.com \\ *通讯作者}

中文摘要. 在当今的液态硝铵储罐系统中, 采用的大多是传统的PID温度控制, 通过控 制加热蒸汽阀的流量来控制液态硝铵储罐 的出口温度。但在其生产过程中, 存在的诸 多扰动, 加上被控对象具有的非线性、慢时 变和大滞后等特点, 单一的PID控制方法已 不能很好的对温度进行精确的控制。而在整 个生产线的运行过程中, 又要保持生产过程 中温度的相对稳定, 同时减少在生产过程中 的能源过度消耗, 针对这种情况, 采用一种
新的控制方法对液态硝铵储罐进行温度控 制是十分必要的。模糊参数自整定PID可以 根据温度偏差及其变化率的不同, 选择不同 的P、I、D参数对系统进行控制, 进而达到 在在线自整定的功能, 以便更好的用于生产 线的运行。

\section{关键词: 液态硝铵储罐; 模糊自整} 定：PID;温度控制 


\section{1. 引言}

液态硝酸铵溶液直接用于生产乳化炸 药的技术如今已在国内的生产厂家中得到 大力的推广, 该技术在生产时在简化生产工 艺、优化设备、降低成本、提高系统的安全 性等方面具有很好的优势。但由于硝酸铵溶 液具有很高的浓度和温度, 为防止析晶, 需 要不断的加热和搅拌, 因此必须对液态硝铵 溶液储罐进行温度控制以满足生产的需求。 目前, 传统的温度控制方式常采用PID控 制, 但是由于液态硝酸铵储罐罐体的数学模 型的还不能精确建立、被控对象本身的非线 性、慢时变、大滞后的特点。传统的PID控 制系统已不能满足需求, 因此有必要采用一 种更为先进的控制策略。

\section{2. 液态硝铵储罐温度控制系统}

常用的液态硝铵储罐由于储罐较高、体 积较大、罐内液体温度分布不均匀等原因, 设置通常设计为两段加温模式。如图1所示, 通常在罐体的不同高度设置温度传感器与 加温控制阀, 以控制和掌握罐体内不同高度 范围内的温度。

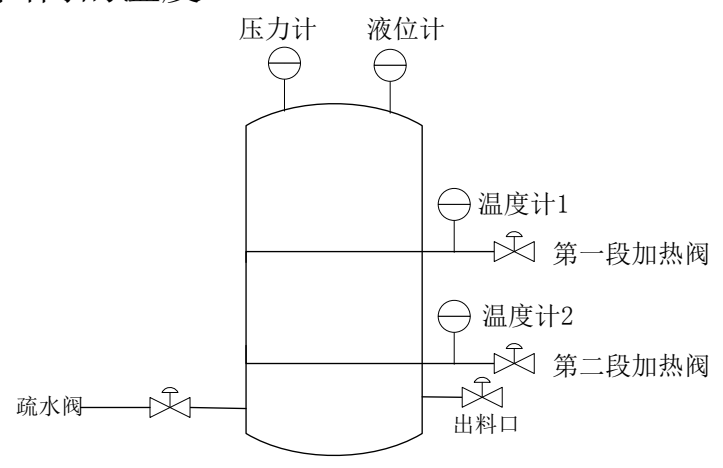

图1 液态硝铵储罐

\section{3. 液态硝铵储罐温度控制系统}

对于液态硝铵储罐系统的温度控制, 就 是对液态硝铵储罐中硝铵水溶液的出口温 度进行自动控制调节。在传统的PID控制 中, 参数的调节往往经过人工整定后便无法 改变, 这样在遇到干扰时便无法很好的控制 被控对象。在面对非线性、慢时变、大滞后 等问题时，传统的PID控制已无法胜任。

模糊自整定参数PID控制器便是针对 此种情况应运而生的, 它结合了常规的PID
控制与模糊控制, 并在计算机硬件平台上得 以实现。首先运用模糊数学方法, 建立模糊 控制规则, 然后将控制规则和有关信息作为 知识库输入计算机中, 最后计算机根据实时 的动态响应情况, 运用模糊推理即可实现对 PID参数的在线自整定。控制系统主要控制 框图如图2所示

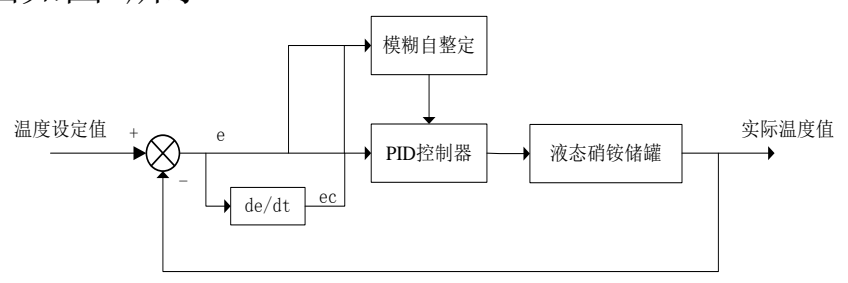

图2 模糊自整定PID控制结构图

\section{4. 模糊自整定PID控制器控制器的设计}

\section{1 量化因子的确定}

本文以液态硝铵储罐温度控制位研 究对象, 通过温度传感器采集工业现场 的实际温度, 与设定值进行比较, 得到 偏差 $e$ 以及其温度变化率 $e c$, 将这两者 作为模糊自适应的PID控制器的输入。得 到 $\{N B, N M, N S, Z E, P S, P M, P B\}$ 的模糊集， 系统控制输出变量 $K_{p} 、 K_{I} 、 K_{d}$ 的模糊集 取 $\{Z E, S, M, B, V B\}$. 其中 $e$ 和 $e c$ 的模糊论 域取: $\{-3,3\}$, 输出变量 $K_{p} 、 K_{I} 、 K_{d}$ 论域 为 $\{0,8\}$, 模糊控制器设计如图3所示, 系统设计为具有 2 输入, 3 输出, 共 49 条 模糊规则。其MATLAB的动态仿真环境图 如图4。

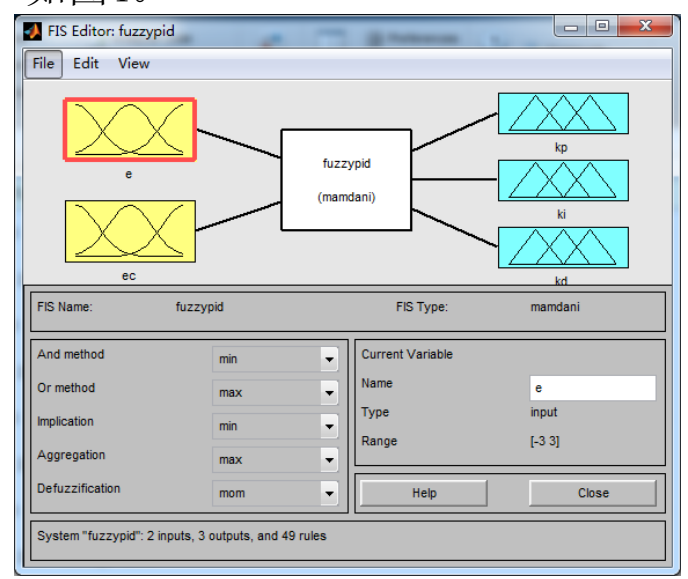

图3 模糊控制器设计 


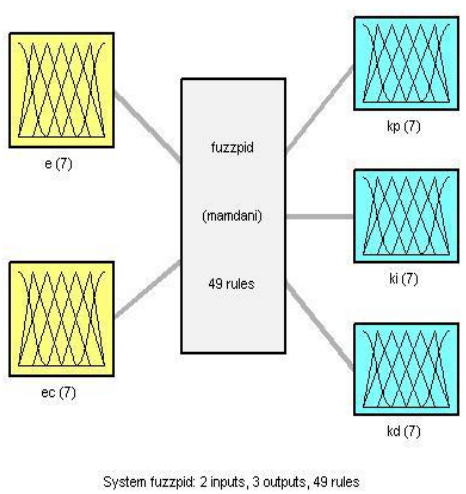

图4 动态仿真环境

\section{2 模糊规则控制表}

PID参数的设置要综合考虑 $K_{p} 、 K_{I} 、 K_{d}$ 三个参数的综合作用, 只有结合实际的工程 经验和控制理论基础，才能建立好合适的、 准确的模糊控制表。本文将偏差 $e$ 以及其温 度变化率 $e c$ 作为系统的输入, 将 $K_{p} 、 K_{I} 、 K_{d}$ 作为控制器的输出, 组成二输入三输出的模 糊控制器, 设计出 49 条模糊规则, 最终的模 糊规则表如表1所示。

\section{3 模糊自整定PID控制器控制器与传统 PID的仿真比较}

根据设计的模糊规则, 在MATLAB中 对模糊自整定PID进行仿真，其仿真曲线如 图5所示。同时在 $2 \mathrm{~s}$ 时, 对系统加入一个扰 动, 由图可知模糊自整定PID控制器与传统 PID控制相比, 其抗干扰能量、响应速度、 调节精度、稳态性能、鲁棒性均优于传统的 PID控制, 特别是对复杂的系统可以获得令 人满意的控制效果, 可以很好的适合现实的 工业生产中。

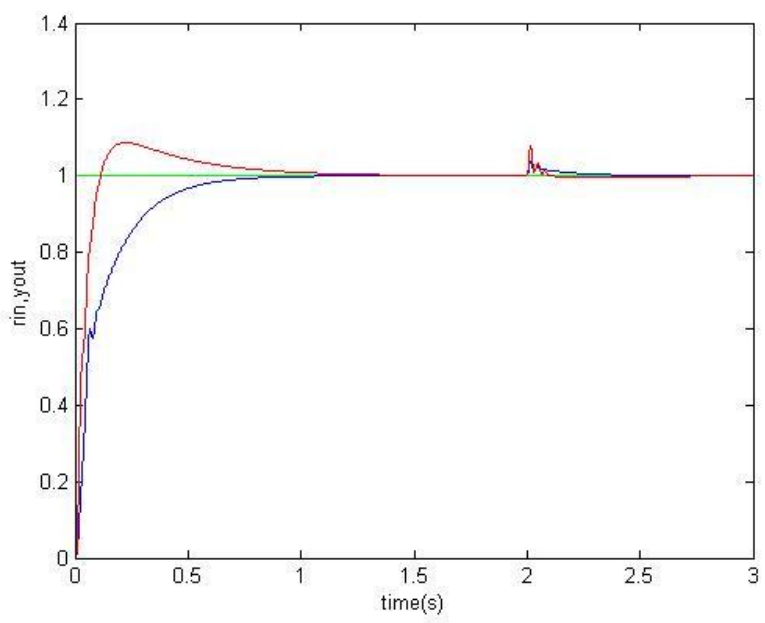

图5 仿真结果曲线

表 1 模糊规则表

\begin{tabular}{|c|c|c|c|c|c|c|c|c|}
\hline \multirow{2}{*}{\multicolumn{2}{|c|}{$K_{p} 、 K_{I} 、 K_{d}$}} & \multicolumn{7}{|c|}{$e c$} \\
\hline & & NB & NM & $\mathrm{NS}$ & $\mathrm{ZE}$ & PS & PM & $\mathrm{PB}$ \\
\hline \multirow{7}{*}{$e$} & NB & VB/ZE/ZE & $\mathrm{VB} / \mathrm{ZE} / \mathrm{ZE}$ & VB/ZE/ZE & VB/ZE/S & VB/ZE/ZE & VB/ZE/VB & $\mathrm{VB} / \mathrm{ZE} / \mathrm{VB}$ \\
\hline & NM & VB/ZE/ZE & VB/ZE/ZE & VB/ZE/ZE & $\mathrm{VB} / \mathrm{ZE} / \mathrm{S}$ & $\mathrm{VB} / \mathrm{ZE} / \mathrm{M}$ & $\mathrm{VB} / \mathrm{ZE} / \mathrm{B}$ & $\mathrm{VB} / \mathrm{ZE} / \mathrm{B}$ \\
\hline & NS & B/ZE/ZE & B/ZE/ZE & B/ZE/ZE & B/ZE/ZE & $\mathrm{B} / \mathrm{ZE} / \mathrm{S}$ & $\mathrm{B} / \mathrm{ZE} / \mathrm{M}$ & $\mathrm{B} / \mathrm{ZE} / \mathrm{B}$ \\
\hline & ZE & $\mathrm{M} / \mathrm{M} / \mathrm{M}$ & $\mathrm{M} / \mathrm{S} / \mathrm{M}$ & $\mathrm{M} / \mathrm{S} / \mathrm{S}$ & M/ZE/ZE & M/ZE/ZE & S/M/ZE & M/S/ZE \\
\hline & PS & $\mathrm{S} / \mathrm{S} / \mathrm{B}$ & $\mathrm{S} / \mathrm{M} / \mathrm{S}$ & $\mathrm{S} / \mathrm{M} / \mathrm{S}$ & M/M/ZE & $\mathrm{M} / \mathrm{M} / \mathrm{S}$ & $\mathrm{M} / \mathrm{M} / \mathrm{M}$ & $\mathrm{M} / \mathrm{B} / \mathrm{B}$ \\
\hline & PM & $\mathrm{B} / \mathrm{M} / \mathrm{ZE}$ & $\mathrm{B} / \mathrm{B} / \mathrm{ZE}$ & $\mathrm{B} / \mathrm{B} / \mathrm{ZE}$ & $\mathrm{B} / \mathrm{B} / \mathrm{ZE}$ & $\mathrm{B} / \mathrm{B} / \mathrm{S}$ & $\mathrm{B} / \mathrm{B} / \mathrm{S}$ & $\mathrm{VB} / \mathrm{VB} / \mathrm{M}$ \\
\hline & PB & $\mathrm{VB} / \mathrm{VB} / \mathrm{ZE}$ & $\mathrm{VB} / \mathrm{VB} / \mathrm{ZE}$ & $\mathrm{VB} / \mathrm{VB} / \mathrm{ZE}$ & $\mathrm{VB} / \mathrm{VB} / \mathrm{ZE}$ & $\mathrm{VB} / \mathrm{VB} / \mathrm{ZE}$ & $\mathrm{VB} / \mathrm{VB} / \mathrm{ZE}$ & $\mathrm{VB} / \mathrm{VB} / \mathrm{ZE}$ \\
\hline
\end{tabular}

\section{5. 液态硝铵储罐模糊自整定PID温度控制 在PLC上的实现}

在本文中, 由于生产现场采用的是西门 子PLC作为下位机控制系统, 可通过STL语 言对其进行编程。具体的工艺流程如下: 通 过温度传感器所采集的实际温度值, 经过 A/D转换传送到PLC、PLC计算出温度偏差 $e$, 并偏差值传送到模糊控制器, 模糊控制 器再根据模糊规则计算出 $K_{p} 、 K_{I} 、 K_{d}$, 并将
值传送到PLC的PID控制模块FB41中，完成 控制作用。

\section{1 硬件组态及参数设置}

西门子系列 PLC 编程软件采用的是 SIMATIC STEP7。在设置PLC时, 需要首先 进行硬件设置。在项目下生成一个站点后, 进入硬件组态界面, 按照现场的实际安装模 块和次序, 依次插入机架、电源、CPU、通 信模块、 $\mathrm{I} / \mathrm{O}$ 模块等各模块。随后进入 $\mathrm{CPU}$ 属性窗口, 设置MPI地址 (设定为2) 等。打 开模拟量输入输出模块, 进行模拟量参数设 置: 测量类型为 $4 \mathrm{DMU}$, 测量范围为 
4-20mA。打开模拟量输出模块, 进行模拟 量输出设置, 设置参数类型为 $\mathrm{I}$, 输出范围 为 $4-20 \mathrm{~mA}$ 。模拟量参数设置如图6所示。另 外, 安装模拟量输入输出模块前应该选择量 程卡, 对于输入信号其量程卡类型应该选择 为 $\mathrm{C}$ 。

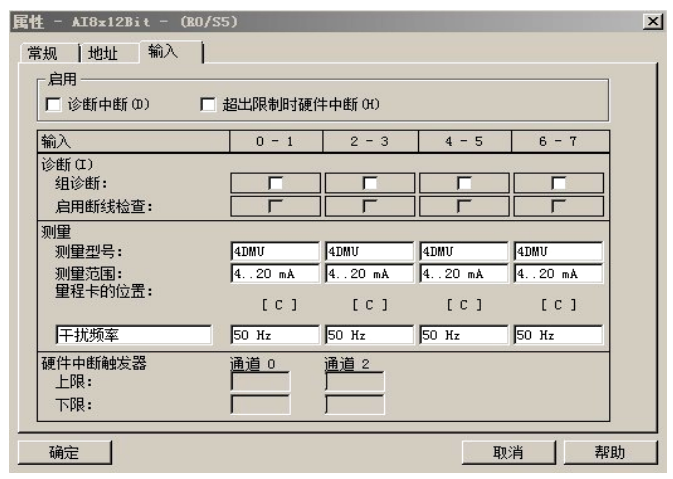

图6模拟量输入模块参数设置

\section{2 模糊参数自整定PID的调用与参数设定}

在西门子PLC系列中, 模糊参数自整定 PID 是在OB35模块中实现的, 同时在运行之 前, 应设置循环中断即采样时间, 目的是使 系统每隔0.1s进行一次运算, 如图7所示。

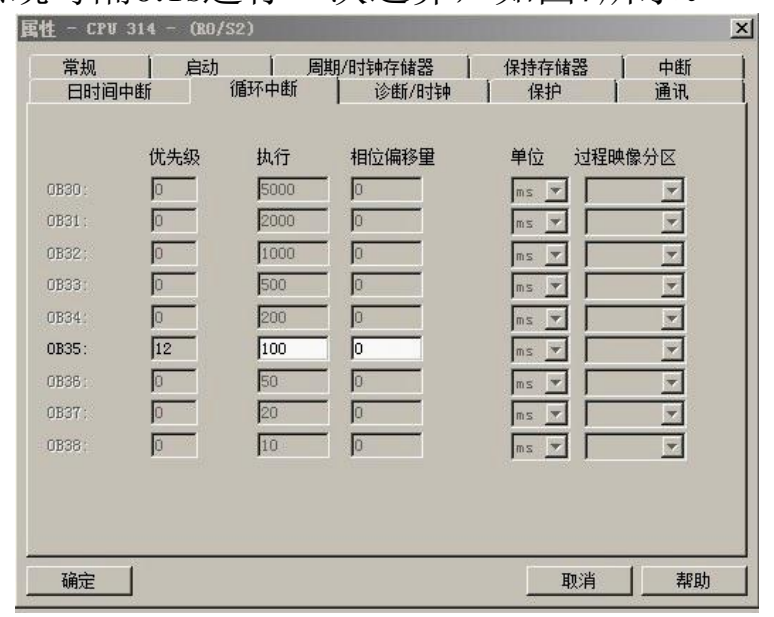

图7 0B35模块采样时间设置

在程序运行之前，应将模糊控制表离线 写入共享数据模块中, 其数据类型应与FB41 数据类型保持一致。在设置完成后, 便可实 现参数自整定PID控制器, 其控制流程图如 图8所示

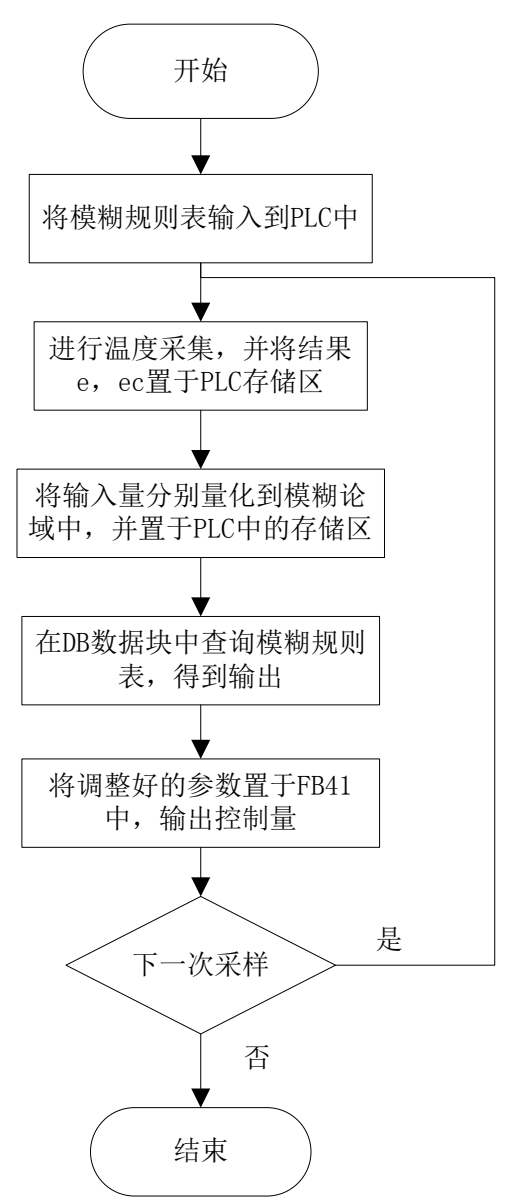

图8 模糊自整定PID参数实现流程图

\section{3 监控软件与运行结果}

在本控制系统中, 可以通过加入嵌入式 MCGS触摸屏, 来实现对现场运行状况实时 监控与控制。在组态界面中, 系在统功能界 面设置了参数设置、历史数据、报警记录等 更为方便的进行远程监控。运行界面如图9 所示。

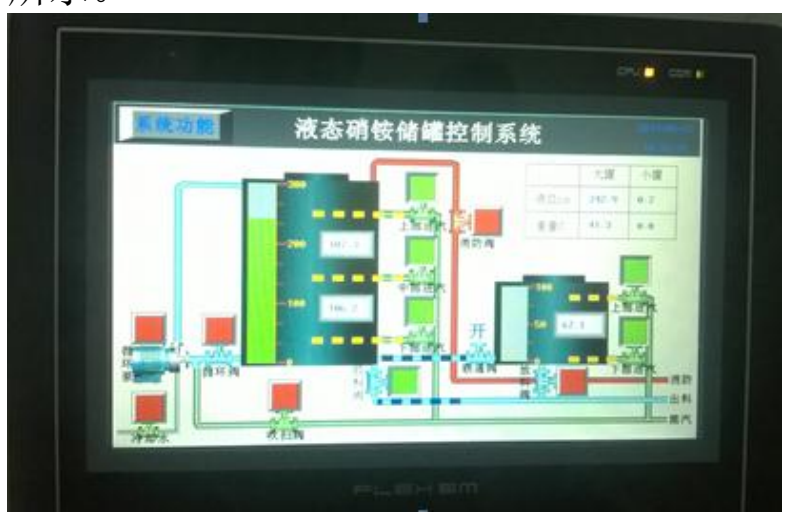

图9 触摸屏运行界面

在系统运行时, 在液态硝铵储罐的出口 出对温度进行测量, 将温度值记录下来汇集 到表格2中。 
表2: 温度控制系统变化

\begin{tabular}{ccc} 
时间 & 出口温度 & 生产状况 \\
\hline $8: 00$ & 96.2 & 开机运行 \\
$9: 00$ & 97.5 & 增加产能 \\
$10: 00$ & 98.1 & 增加产能 \\
$11: 00$ & 99.3 & 增加产能 \\
$12: 00$ & 96.5 & 保温阶段 \\
$13: 00$ & 97.3 & 增加产能 \\
$14: 00$ & 98.2 & \\
$15: 00$ & 99.1 & 增加产能 \\
& & \\
\hline
\end{tabular}

从表 2 中可以看出, 温度控制系统应随 着生产状况的变化, 不断的对液态硝铵储罐 的出口处温度进行调节, 温度控制在95-105 度之间, 在运行过程其温度浮动范围较小, 其未发生超温故障, 达到了良好的控制效 果。

\section{6. 结束语}

本文介绍了工业液态硝铵储罐模糊自 整定PID温度控制设计, 在实际系统的运行 过程中，应用模糊自整定PID控制的液态硝 铵储罐的温度控制效果良好, 到达了预期的 温度控制要求, 统在稳定性、抗干扰性、鲁 棒性均优于与传统的PID控制。特别是在抗 干扰能力方面, 有很好的控制效果, 能够很 好的适应现实生产过程中的控制要求。

\section{References}

[1] Yang Minggang,Xia Guang and Li Fenglei,Design of the Storage and Transportation Technology for Liquid Ammonium Nitrate and Storage Tank,
Explosive

Materials,vol.41, pp.16-19,2012.

[2] John S Manka.Factors that affect the chemical gassing of emulsion explosive. 30th annual conference on explosives and blasting technique . 2004 A. Banerjee, A joint economic-lot-size model for purchaser and vendor, Decision Sciences, vol.17, pp. 292-311, 1986.

[3] LIU Yuxing, Effect of Lowering the Crystallization Point of Liquid Ammonium Nitrate used for Industrial Explosives,Minig $R \& D, \quad$ vol.32, $\mathrm{pp}$. 114-116,2012

[4] Qiao Xiuquan,Application of Fuzzy Control Technology on the liquid state Ammonium Nitrate Storage Tank, Coal Mine Blasting, vol.102, pp. 35-38, 2013.

[5] Zeng Jing and Xie Youcheng, Design of fresh steam temperature cascade control system based on self-tuning PID, Microcomputer Information, vol. 23, pp. 46-48, 2007.

[6] Wen Zejun, Yao Qunyong, and Song Shaojian, Application of one Machine with Multiple Screen in the Control System of Liquid Ammonium Nitrate Storage, Automation \& Instrumentation,vol. 185, pp. 57-60, 2013.

[7] Mahseredjian J, Alvarado F, Creating an electro-magnetic transients program in Matlab, IEEE Tran on Power Delivery, vol. 81, pp. 380-388, 2004.

[8] $\mathrm{Xu}$ Dean, Benefit Analysis on the Application of Ammonium Nitrate Solution Directly in the Industry Explosive Production, Explosive Materials, vol. 40, pp.32-34, 2011. 